Case Report

\title{
Late-Onset Posttransplant Lymphoproliferative Disorder Results in Jejunal Stricture Managed with Endoscopic Dilation
}

\author{
Steven Park (D), ${ }^{1}$ John Miller, ${ }^{2}$ Janice Cheong, ${ }^{2}$ Asad Ullah, ${ }^{2}$ Lawrence Saubermann, ${ }^{2}$ \\ and Arthur Decross ${ }^{2}$ \\ ${ }^{1}$ Department of Internal Medicine, University of Rochester Medical Center, Rochester, NY, USA \\ ${ }^{2}$ Division of Gastroenterology \& Hepatology, University of Rochester Medical Center, Rochester, NY, USA \\ Correspondence should be addressed to Steven Park; steven_park@urmc.rochester.edu
}

Received 12 January 2021; Accepted 31 July 2021; Published 4 August 2021

Academic Editor: Yoshihiro Moriwaki

Copyright (C) 2021 Steven Park et al. This is an open access article distributed under the Creative Commons Attribution License, which permits unrestricted use, distribution, and reproduction in any medium, provided the original work is properly cited.

Background. Late-onset posttransplant lymphoproliferative disorder (PTLD) after orthotopic heart transplantation is rare. Case Presentation. We present a rare diagnosis of small bowel stricture caused by healed lymphomatous ulcers in a patient with orthotopic heart transplantation and PTLD diagnosed 25 years after initial transplantation. We also demonstrate successful endoscopic balloon dilations that improved the patient's obstructive symptoms. Conclusion. It is important to consider stricture from healed lymphomatous ulcers in posttransplant patients presenting with obstructive symptoms.

\section{Background}

Late-onset posttransplant lymphoproliferative disorder (PTLD) after orthotopic heart transplantation is rare. We present a rare diagnosis of small bowel stricture caused by healed lymphomatous ulcers in a patient with orthotopic heart transplantation and PTLD diagnosed 25 years after initial transplantation. We also demonstrate successful endoscopic balloon dilations that improved the patient's obstructive symptoms.

\section{Case Presentation}

A 31-year-old female with a past medical history of two orthotopic heart transplants (at age six for viral cardiomyopathy and at age thirteen for graft vasculopathy) complicated by posttransplant lymphoproliferative disorder (PTLD) (monomorphic with germinal center B-cell type; EBV-negative) presented to our hospital with two weeks of postprandial emesis. Nausea and emesis occurred two to four hours after meals and consisted of nonbloody, nonbilious emesis. Bloodwork was notable for hemoglobin of $8 \mathrm{~g} / \mathrm{dL}$ (11.2-15.7 g/dL). 6 days prior to admission, she had received third cycle of triweekly chemotherapy with cyclophosphamide, doxorubicin, vincristine, and prednisone (CHOP).

Upper endoscopy performed 1 month prior to admission to evaluate for a source of anemia demonstrated scattered, large, nonbleeding, clean-based ulcers in the proximal jejunum and a segment of circumferential ulceration. Biopsies of the jejunal ulcers confirmed involvement by patient's known monomorphic-type PTLD (germinal center B-cell type; EBV-negative). To evaluate for gastric outlet obstruction (GOO) suggested by CT scan in the setting of nausea and emesis, we performed an upper endoscopy. This endoscopy revealed a wide-open pylorus without GOO, and no endoscopic abnormality was seen through the fourth portion of the duodenum. Duodenal biopsies were negative for cytomegalovirus, adenovirus, and herpes simplex. Congo red stains were negative for amyloid, and morphologic evidence of lymphoma was absent.

We were suspicious of a more distal site of obstruction and ordered an MR enterography (Figure 1) that revealed a transition point with focal thickening of the proximal jejunum. The decision was made to pursue endoscopic balloon dilation. On push enteroscopy, we encountered an ulcerated stricture with only $2-3 \mathrm{~mm}$ in luminal diameter (Figure 2). 


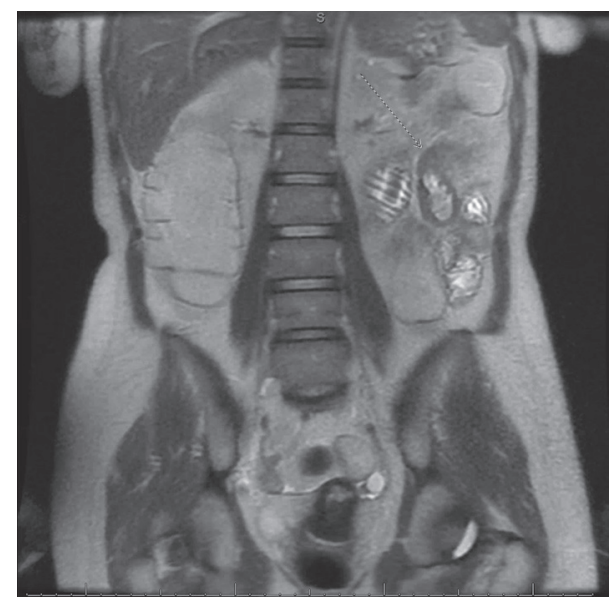

FIGURE 1: MR enterography showing small bowel obstruction with a transition point at focal thickening of the proximal jejunum (arrow).

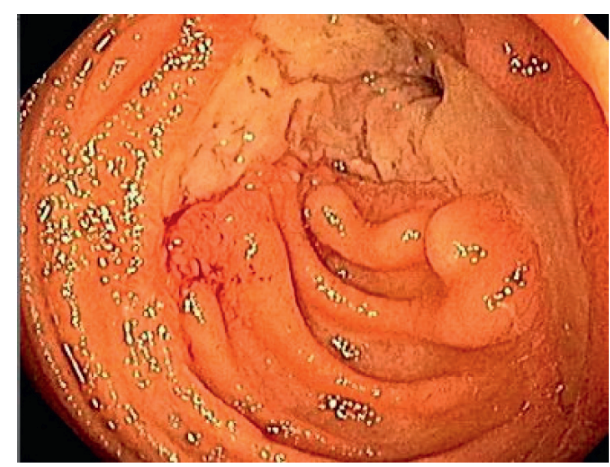

FIGURE 2: EGD showing a clean-based circumferential ulceration in the proximal jejunum with an ulcerated stricture estimated $2 \mathrm{~mm}$ in luminal diameter.

The site of stricture appeared to be the same segment of jejunal ulceration on initial enteroscopy that likely healed with scarring after chemotherapy. Decision was made to proceed with endoscopic dilation using a $6 \mathrm{~mm}$ biliary dilation balloon catheter, the smallest dilation catheter available via long pediatric colonoscope instrument channel. Roughly $4 \mathrm{~mm}$ diameter distention was reached before notable resistance was felt and evidence of trauma was seen. Patient tolerated a full liquid diet postprocedure and was discharged with a plan for a repeat dilation. To date, patient completed two additional endoscopic dilation procedures, followed by a small bowel resection, and remains alive after completion of total 6 rounds of R-CHOP without additional gastrointestinal complications.

\section{Discussion and Conclusions}

PTLD is a heterogeneous lymphoid proliferation that occurs after solid-organ or hematopoietic transplantation in the setting of prolonged immunosuppression [1-3]. The median time to diagnosis after transplantation is 2.5 years, and the incidence of PTLD in cardiac transplant patients ranges from 5 to $9 \%$ [4-6]. The clinical manifestations vary from nonspecific symptoms to sudden enlargement of nodal and extranodal lymphoid organs [7]. Lymphoid proliferations range from benign collections to malignant lymphomas involving T cells, B cells, and/or plasma cells [8]. PTLD is categorized based on the WHO classification of lymphoid neoplasm, and three main stages of evolution are recognized: early, polymorphic, and monomorphic lesions [9]. Polymorphic and monomorphic B-cell PTLD are the two most common histologic subsets, with increasing proportion of monomorphic cases noted in recent decades [8]. Of note, our patient had monomorphic-type PTLD with negative EBV stain, germinal center B-cell type with immunohistochemistry negative for NYC and positive for BCL2.

PTLD can also involve other organs such as central nervous system, bone marrow, spleen, lung, liver, and kidney [7]. PTLD involvement of small bowel in the form of GI bleed and viral infection has been reported previously [10-13]. However, a stricture in the small bowel from healed lymphomatous ulcer causing obstruction and needing endoscopic dilation has not been reported to our knowledge.

Our case highlights a rare diagnosis of small bowel stricture caused by healed lymphomatous ulcers in a patient with orthotopic heart transplantation and PTLD diagnosed 25 years after initial transplantation. We demonstrate successful endoscopic balloon dilations that improved the patient's obstructive symptoms. A multidisciplinary care involving oncology, pathology, gastroenterology, and colorectal surgery service was crucial to addressing gastrointestinal complications of PTLD. It is important to consider stricture from healed lymphomatous ulcers in posttransplant patients presenting with obstructive symptoms.

\section{Abbreviations}

PTLD: Posttransplant lymphoproliferative disorder

CHOP: Cyclophosphamide, doxorubicin, vincristine, and prednisone

GOO: Gastric outlet obstruction

MR: Magnetic resonance.

\section{Data Availability}

No data were used to support this study.

\section{Conflicts of Interest}

The authors declare that they have no conflicts of interest.

\section{References}

[1] D. Hayes, D. Tumin, R. E. Foraker, and J. D. Tobias, "Posttransplant lymphoproliferative disease and survival in adult heart transplant recipients," Journal of Cardiology, vol. 69, no. 1, pp. 144-148, 2017.

[2] C. C. Tai, J. L. Curtis, J. R. Szmuszkovicz et al., “Abdominal involvement in pediatric heart and lung transplant recipients with posttransplant lymphoproliferative disease increases the risk of mortality," Journal of Pediatric Surgery, vol. 43, no. 12, pp. 2174-2177, 2008.

[3] M. J. Aull, J. F. Buell, J. Trofe et al., "Experience with 274 cardiac transplant recipients with posttransplant 
lymphoproliferative disorder: a report from the Israel Penn international transplant tumor registry," Transplantation, vol. 78, no. 11, pp. 1676-1682, 2004.

[4] S. A. Webber, D. C. Naftel, F. J. Fricker et al., "Lymphoproliferative disorders after paediatric heart transplantation: a multi-institutional study," The Lancet, vol. 367, no. 9506, pp. 233-239, 2006.

[5] B. Z. Katz, E. Pahl, S. E. Crawford et al., "Case-control study of risk factors for the development of post-transplant lymphoproliferative disease in a pediatric heart transplant cohort," Pediatric Transplantation, vol. 11, no. 1, pp. 58-65, 2007.

[6] G. E. Wilde, D. J. Moore, and R. D. Bellah, "Posttransplantation lymphoproliferative disorder in pediatric recipients of solid organ transplants: timing and location of disease," American Journal of Roentgenology, vol. 185, no. 5, pp. 1335-1341, 2005.

[7] H. A. H. Ibrahim and K. N. Naresh, "Posttransplant lymphoproliferative disorders," Advances in Hematology, vol. 2012, Article ID 230173, 11 pages, 2012.

[8] D. E. Tsai, S. Bagley, R. Reshef et al., "The changing face of adult posttransplant lymphoproliferative disorder: changes in histology between 1999 and 2013," American Journal of Hematology, vol. 93, no. 7, pp. 874-881, 2018.

[9] S. H. Swerdlow, "Post-transplant lymphoproliferative disorders: a working classification," Current Diagnostic Pathology, vol. 4, no. 1, pp. 28-35, 1997.

[10] A. Siegel, J. Boike, I. Trivedi, and R. Yadlapati, "Posttransplant lymphoproliferative disorder of the small bowel as an unexpected cause of iron deficiency anemia decades after heart transplantation," ACG Case Reports Journal, vol. 4, p. e86, 2017.

[11] D. Nitta, K. Kinugawa, T. Imamura et al., "How should we treat early post-transplant lymphoproliferative disease after heart transplantation?" International Heart Journal, vol. 56, no. 6, pp. 676-678, 2015.

[12] P. Burra, A. Buda, U. Livi et al., "Occurrence of post-transplant lymphoproliferative disorders among over thousand adult recipients: any role for hepatitis C infection?" European Journal of Gastroenterology \& Hepatology, vol. 18, no. 10, pp. 1065-1070, 2006.

[13] U. Allen and J. Preiksaitis, "Post-transplant lymphoproliferative disorders, epstein-barr virus infection, and disease in solid organ transplantation: guidelines from the American society of transplantation infectious diseases community of practice," Clinical Transplantation, vol. 33, no. 9, Article ID e13652, 2019. 